\title{
Identification of thyroid hormone residues on serum thyroglobulin: a clue to the source of circulating thyroglobulin in thyroid diseases
}

\author{
Laure Druetta ${ }^{1}$, Hubert Bornet ${ }^{1,2}$, Geneviève Sassolas ${ }^{3}$ and Bernard Rousset ${ }^{1,4}$ \\ ${ }^{1}$ Institut National de la Santé et de la Recherche M édicale, U nité 369, Faculté de M édecine Lyon-RTH Laënnec, 69372 Lyon, France, \\ ${ }^{2}$ Laboratoire de Biophysique, Faculté de M édecine Lyon-Grange Blanche, 69008 Lyon, France, ${ }^{3}$ Département de M édecine N ucléaire, Hôpital Louis \\ Pradel, 69394 Lyon, France and ${ }^{4} U$ nité Fonctionnelle de Biologie Cellulaire, Fédération de Biochimie, Hôpital Edouard Herriot, 69437 Lyon, France \\ (Correspondence should be addressed to B Rousset, IN SERM U 369, Faculté de M édecine Lyon-RTH Laënnec, 69372 Lyon Cedex 08 , France)
}

\begin{abstract}
Thyroglobulin (Tg) present in the serum of normal individuals and patients with thyroid disorders could be partly newly synthesized non-iodinated Tg and partly Tg containing iodine and hormone residues originating from the lumen of thyroid follicles. With the aim of examining the contribution of the latter source of $\mathrm{Tg}$ to the elevation of serum $\mathrm{Tg}$ concentration in thyroid pathophysiological situations, we devised a procedure to identify thyroxine (T4) and tri-iodothyronine (T3) residues on $\mathrm{Tg}$ from unfractionated serum. A two-step method, based on (i) adsorption of Tg on an immobilized antihuman $\mathrm{Tg}(\mathrm{hTg})$ monoclonal antibody $(\mathrm{mAb})$ and (ii) recognition of hormone residues on adsorbed $\mathrm{Tg}$ by binding of radioiodinated anti-T4 $\mathrm{mAb}$ and anti-T3 $\mathrm{mAb}$, was used to analyze serum $\mathrm{Tg}$ from patients with either Graves' disease (GD), subacute thyroiditis (ST) or metastatic differentiated thyroid cancer (DTC). Purified hTg preparations with different iodine and hormone contents were used as reference. Adsorption of purified Tg and serum Tg on immobilized anti-hTg mAb ranged between 85 and $90 \%$ over a wide concentration range. Labeled anti-T4 and anti-T3 mAbs bound to adsorbed purified Tg in amounts related to its iodine content. Tg adsorbed from six out of six sera from ST exhibited anti-T4 and anti-T3 mA binding activities. In contrast, significant mAb binding was only observed in one out of eight sera from untreated GD patients and in 1 out of 13 sera from patients with DTC. The patient with DTC, whose serum Tg contained T4 and T3, represented a case of hyperthyroidism caused by a metastatic follicular carcinoma. In conclusion, we have identified, for the first time, $\mathrm{T} 4$ and $\mathrm{T} 3$ residues on circulating $\mathrm{Tg}$. The presence of $\mathrm{Tg}$ with hormone residues in serum is occasional in GD and DTC but is a common and probably distinctive feature of ST.
\end{abstract}

European Journal of Endocrinology $140457-467$

\section{Introduction}

Thyroglobulin $(\mathrm{Tg})$, the thyroid hormone precursor protein, is normally stored in the lumen of thyroid follicles. In this compartment, Tg undergoes chemical reactions (iodination and coupling of tyrosyl residues) leading to the formation of thyroid hormone residues (thyroxine (T4) and tri-iodothyronine (T3)) within its polypeptide backbone. In the normal human thyroid gland, the hormone content of $\mathrm{Tg}$ ranges from one to three T4 or T3 residues per molecule (1), T3 amounting to from one-fifth to one-twentieth of T4. In normal individuals, the thyroid gland secretes low amounts of $\mathrm{Tg}$ into the circulation, but in diverse pathophysiological situations serum $\mathrm{Tg}$ concentration markedly increases. $\mathrm{Tg}$ molecules appearing in the circulation of normal subjects or patients with thyroid disorders probably originate from two distinct thyroid compartments. First, newly synthesized Tg molecules (devoid of hormone residues), instead of being secreted into the lumen of follicles, could be released at the basolateral membrane of thyrocytes (2) into the extrafollicular or interstitial fluid. Secondly, iodinated Tg molecules (with hormones residues) internalized at the apical pole of thyrocytes from the follicle lumen could undergo a transcellular vesicular transport or transcytosis (3) to be released into the extrafollicular fluid. The elevation of serum $\mathrm{Tg}$ concentration in response to thyroid stimulation by thyrotropin (TSH) $(4,5)$, or by anti-TSH receptor stimulating antibodies (6) in patients with Graves' disease (GD), could result from the activation of either of these pathways, or both of them. In pathological situations leading to morphological alterations of the thyroid paren chyma, as seems to be the case in subacute thyroiditis (ST), the appearance of $\mathrm{Tg}$ in the blood stream might result from the leakage of $\mathrm{Tg}$ from 
disrupted thyroid follicles. The mode of release of $\mathrm{Tg}$, sometimes in very large amounts, into the circulation of patients with metastatic differentiated thyroid cancer (DTC) is more hypothetical. It certainly depends on the organization of thyrocytes within the tumor. When present, follicular structures are probably subjected to intense remodeling due to cytolysis and/or cell proliferation that could lead to the release of their $\mathrm{Tg}$ content. If tumor cells are not organized in follicles, newly synthesized $\mathrm{Tg}$ would be released as a consequence of the lack of a storage compartment. Thus, according to the nature of the thyroid disease and the structural and functional alterations of thyroid tissue, the compartment from which $\mathrm{Tg}$ is released and the release process are likely to be distinct. To identify serum $\mathrm{Tg}$ molecules originating from the lumen of thyroid follicles that have undergone hormone synthesis reactions, we devised a procedure of detection of T4 and T3 residues on low amounts of $\mathrm{Tg}$ in complete serum. A two-step method based on (i) the adsorption of $\mathrm{Tg}$ on an immobilized anti-human $\mathrm{Tg}(\mathrm{hTg})$ monoclonal antibody (mAb) and (ii) the recognition of T4 and T3 residues on adsorbed $\mathrm{Tg}$ by binding of radioiodinated anti-T4 and anti-T3 mAbs, was used to analyze serum $\mathrm{Tg}$ from patients with different thyroid disorders (GD, ST or DTC).

\section{Materials and methods}

\section{Materials}

Anti-T4 and anti-T3 mAbs and the immobilized antihTg $\mathrm{mA} b$ were obtained from Cis bio international (Gifsur-Yvette, France). The anti-T4 mA $b$ and anti-T3 mA $b$ were designated R41 and R30 respectively. The antihTg mAb, immobilized on plastic tubes with gills, was the antibody used in the ELSA-HTG Kit commercialized by Cis bio international. $\left[{ }^{125} \mathrm{I}\right] \mathrm{T} 4$ and $\left[{ }^{125} \mathrm{I}\right] \mathrm{T} 3$ (specific radioactivity $>1200 \mu \mathrm{Ci} / \mu \mathrm{g}$ ) were purchased from Amersham Pharmacia Biotech, Orsay, France. Sheep anti-mouse IgG immobilized on activated silica beads was obtained from Biogenesis (Poole, Dorset, UK).

\section{hTg preparations}

Tg was purified from fresh human thyroid tissue from euthyroid or hypothyroid patients with goiter. To minimize potential Tg degradation, thyroid tissue was minced into small pieces and $\mathrm{Tg}$ leaking from open follicles was collected in $0.01 \mathrm{~mol} / \mathrm{l}$ sodium phosphate, $0.15 \mathrm{~mol} / \mathrm{l} \mathrm{NaCl}, \mathrm{pH} 7.4$ (PBS) for $16 \mathrm{~h}$ at $4{ }^{\circ} \mathrm{C}$. Tg in solution was precipitated by $1.4-1.8 \mathrm{~mol} / \mathrm{l}$ ammonium sulfate. Precipitated protein, collected by centrifugation at $55000 \mathrm{~g}$ for $20 \mathrm{~min}$ at $4{ }^{\circ} \mathrm{C}$, was solubilized and dialyzed against the same buffer. $\mathrm{Tg}$ was purified by anion-exchange chromatography on DEAE-Sepharose and gel filtration on Sepharose CL-4B (Pharmacia LKB, Uppsala, Sweden). The Tg concentration was assayed by absorbance measurement at $280 \mathrm{~nm}(\mathrm{e}=660000$ per $\mathrm{mol} / \mathrm{l}$ per $\mathrm{cm}$ ) and by the Bradford method using BSA as standard. Three hTg preparations, hTgRP3, hTgRP4 and hTgRP8 were used in this study. The structural integrity of purified Tg was checked by SDS-PAGE. In non-reducing conditions, the three $\mathrm{Tg}$ preparations gave a single band representing $\mathrm{Tg}$ monomers. In reducing conditions, two bands were observed; they correspond to the ' $\mathrm{S}$ ' and ' $\mathrm{F}$ ' bands described by Gentile et al. (7). The iodine content of hTgRP3, hTgRP4 and hTgRP8, determined by the method of Sandell \& Kolthoff (8) using a Technicon AutoA nalyser (Technicon, Palo Alto, CA, USA), was respectively 8, 2 and 20 iodine atoms per Tg molecule. The T4 content of hTgRP3 and hTgRP8 (determined by T4 RIA after pronase hydrolysis) was about 1.0 and 2.1 residues per Tg molecule respectively. hTgRP4 was available in too low amounts to allow measurements of its T4 content.

\section{Serum samples}

Sera were obtained from eight patients with GD prior to any treatment. GD was diagnosed on the basis of clinical symptoms of hyperthyroidism and measurements of serum TSH and free T4 concentrations. Immunoassayable serum $\mathrm{Tg}$ concentrations ranged from 120 to $1230 \mathrm{ng} / \mathrm{ml}$ (mean $542 \mathrm{ng} / \mathrm{ml}$ ). Serum Tg concentrations in normal subjects ranged from 2 to $40 \mathrm{ng} / \mathrm{ml}$. Six serum samples were collected from patients with ST diagnosed on clinical and serological grounds including elevated levels of circulating $\mathrm{Tg}(65-3000 \mathrm{ng} / \mathrm{ml}$, mean $732 \mathrm{ng} / \mathrm{ml}$ ). Thirteen sera from patients with DTC were selected for their high serum $\mathrm{Tg}$ concentration (60 to $30000 \mathrm{ng} / \mathrm{ml}$, mean $6593 \mathrm{ng} / \mathrm{ml}$ ). All patients had undergone total thyroidectomy and had received radioiodine for ablation of thyroid remnants. At the time of serum sampling, there was scintigraphic evidence for metastasis in all patients. None of the sera from patients with either GD, ST or DTC contained detectable anti-Tg autoantibodies assessed as previously described (9).

Sera from thyroidectomized patients shown to be devoid of immunoreactive $\mathrm{Tg}$ and detectable anti-Tg autoantibodies were pooled and this pool, designated 'serum So', was used as control or as diluent for patient serum and/or hTgRP samples.

All serum samples were taken from blood specimens sent for routine biochemical analyses; in no case was blood sampling solely for the purpose of the study.

\section{Case report - patient DTC 89}

Patient DTC 89, a 63-year-old man, presented in 1989 with a cervical and endothoracic compressive goiter containing a large zone without radioiodine uptake activity with the association of hyperthyroidism. An extensive cervicomediastinal surgical procedure was performed with removal of a poorly differentiated follicular thyroid cancer exhibiting intravascular 
emboli. Post-operatively, serum free T4 (105 pmol/l) and $\mathrm{Tg}$ levels $(31000 \mathrm{ng} / \mathrm{ml}$ ) were extremely elevated. A whole body scan (WBS), after administration of $100 \mathrm{mCi}^{131}$, disclosed many sites of radioiodine uptake in lungs and liver while there was no uptake in the thyroid bed. These metastases were confirmed by a computerized tomography (CT) scan. Treatment with methimazole was undertaken over 4 months and was withdrawn after the second $100 \mathrm{mCi}$ dose, since euthyroidism was attained. Then the patient received suppressive T4 treatment. Following repeated ${ }^{131}$ I treatments, radioiodine uptake sites progressively declined in number and intensity, and serum $\mathrm{Tg}$ concentration markedly decreased. A fter administration of a total dose of $700 \mathrm{mCi}$, there was no more radioactive iodine uptake. When serum Tg concentration increased again, an exploration with Octreoscan (Mallinckrodt, Petten, the Netherlands) was performed, showing multiple mediastinal metastases, confirmed by CT scan. Finally, the patient died due to extensive metastatic diffusion. In this patient, production of thyroid hormones by hepatic and lung metastases of a follicular cancer is demonstrated by persistence of hyperthyroidism after total thyroidectomy.

\section{Radioiodination of proteins}

Purified Tg, anti-T4 mAb and anti-T3 mAb were labeled with ${ }^{125}$ I using lodogen (Sigma Chemical Co., St Louis, MO, USA) as a solid phase iodinating reagent. Fifty microliters of lodogen $(100 \mu \mathrm{g} / \mathrm{ml})$ in chloroform were used to coat plastic tubes. After evaporation of chloroform, $10 \mu \mathrm{g}$ Tg or $50 \mu \mathrm{g} \mathrm{mAb}$ were mixed with $300 \mu \mathrm{Ci} \mathrm{Na}{ }^{125}$ | (ICN Biomedicals, Orsay, France) in $50 \mu \mathrm{l} \mathrm{PBS}, \mathrm{pH}$ 7.4. After $15 \mathrm{~min}$ at $20^{\circ} \mathrm{C}$, the labeling mixture was fractionated on a G-25 M Sephadex column to separate the ${ }^{125} \mathrm{I}$-labeled protein from free radioiodide. The specific radioactivity was about $25 \mu \mathrm{Ci} / \mu \mathrm{g}$ for $\mathrm{hTg}$ and $3-5 \mu \mathrm{Ci} / \mu \mathrm{g}$ for anti-T4 $\mathrm{mAb}$ and anti-T3 $\mathrm{mAb}$.

\section{Tg RIA}

A rabbit anti-hTg immune serum at a $1: 10^{6}$ final dilution was mixed with $0.1-50 \mathrm{ng}$ purified $\mathrm{Tg}$ (hTgRP8) or 0.1$100 \mu \mathrm{l}$ human serum and $100 \mu \mathrm{l}$ 125/-hTgRP8 (40000 c.p.m.) in a total volume of $400 \mu \mathrm{l}$ PBS containing $2 \mathrm{mg} / \mathrm{ml}$ BSA. Incubation was carried out for $16 \mathrm{~h}$ at room temperature. Immune complexes were collected using sheep anti-rabbit immunoglobulinsimmobilized on activated silica beads (Biogenesis). A fter 30 min incubation at room temperature, beads were pelleted by centrifugation at $1500 \mathrm{~g}$ for $30 \mathrm{~min}$ at $4^{\circ} \mathrm{C}$, washed in PBS-BSA and the radioactivity measured using a gamma counter. Each serum sample was assayed at two or three dilutions made in duplicate. The sensitivity of the assay was about $2 \mathrm{ng} / \mathrm{ml}$. The interassay coefficient of variation was $10-15 \%$. Comparison of reference $\mathrm{Tg}$ with the international $\mathrm{hTg}$ reference material (CRM 457) (10) showed a 1.2 factor of difference.

\section{Procedure to detect thyroid hormone residues on Tg}

Adsorption of Tg Serum samples (from $30 \mu \mathrm{l}$ to $1 \mathrm{ml}$, supplemented to $1 \mathrm{ml}$ with serum So) from patients with either GD, ST or DTC, or purified Tg (hTgRP3, hTgRP4 or hTgRP 8: 10-1000 ng diluted in $1 \mathrm{ml}$ serum So) were introduced into anti-hTg mAb-coated tubes. After incubation for $6 \mathrm{~h}$ at $20^{\circ} \mathrm{C}$ and overnight at $4{ }^{\circ} \mathrm{C}$, serum was removed and its $\mathrm{Tg}$ content was assayed by RIA. The difference between the amount of immunoassayable serum $\mathrm{Tg}$ before and after adsorption gave the amount of bound or adsorbed $\mathrm{Tg}$.

\section{Hormone residue recognition on adsorbed $\mathrm{Tg}$ by} anti-T4 mAb or anti-T3 mAb binding assay After two washings with PBS-Tween $0.05 \%$ to remove any residual serum components, adsorbed Tg was allowed to react with ${ }^{125}$ I-labeled anti-T4 $\mathrm{mAb}$ or ${ }^{125}$ /-labeled anti-T3 mAb (about 100000 c.p.m., i.e. $15 \mathrm{ng}$ or $0.1 \mathrm{pmol}$ ) in a total volume of $1 \mathrm{ml}$ PBS-BSA. After incubation overnight at $20^{\circ} \mathrm{C}$, unbound radioactivity was removed and tubes were washed three times with PBS-Tween $0.05 \%$. Radioactivity was measured using a gamma counter. Each serum sample was analyzed at least in duplicate.

\section{Results}

\section{Characterization of the anti-T4 and anti-T3 mAbs and analysis of their capacity to react with hormone residues on $\mathrm{Tg}$ in solution}

Figure $1 \mathrm{~A}$ shows the hormone binding activity of increasing amounts of the anti-T4 $\mathrm{mAb}$ and anti-T3 $m A b$. The amount of $m A b$ that bound $30 \%$ of the tracer was $30 \mathrm{ng}$ for anti-T4 mA $b$ and $10 \mathrm{ng}$ for anti-T3 mA . These amounts of $\mathrm{mAb}$ were used to analyze the $T 4, \mathrm{~T} 3$ and reverse tri-iodothyronine (rT3) binding specificity in competitive binding experiments (Fig. $1 \mathrm{~B}$ and $\mathrm{C}$ ). The T4 concentration that produced a $50 \%$ decrease of the binding of $\left.{ }^{125} I\right] T 4$ to the anti-T4 mAb was about $8 \mathrm{nmol} / \mathrm{l}$. T3, up to $1 \mu \mathrm{mol} / \mathrm{l}$, had a very limited or no displacing activity. rT3 inhibited labeled T4 binding but at concentrations 100 -fold higher than those of T4. The T3 concentration that produced a 50\% decrease of the binding of $\left[{ }^{125} \mathrm{I}\right] \mathrm{T} 3$ to the anti-T3 mAb was about $3 \mathrm{nmol} / \mathrm{l}$. rT3 did not displace labeled T3 binding. T4 competed with labeled T3 for binding to the anti-T3 $\mathrm{mAb}$ but at concentrations more than 100-fold higher than those of T3. Thus, each of the two mA bs exhibited a rather high apparent affinity and a good specificity for its antigen, either T4 or T3.

We examined whether anti-T4 mAb and anti-T3 $\mathrm{mA} b$ were capable of detecting hormone residues on $\mathrm{Tg}$ 

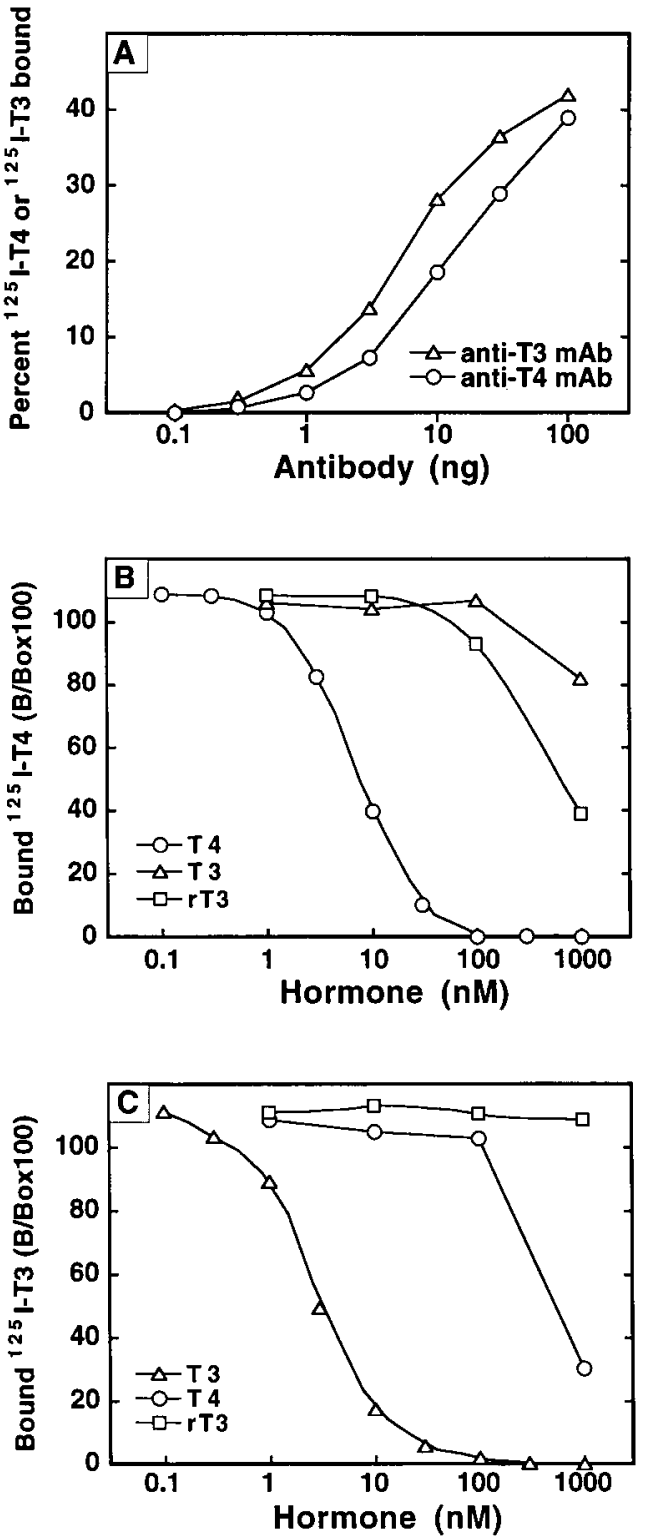

Figure 1 Characterization of the anti-T4 and anti-T3 mAbs. (A) Hormone binding activity of mAbs. $\left[{ }^{125}\right.$ I]T4 or $\left[{ }^{125}\right.$ I]T3

$(\approx 40000$ c.p.m.) was incubated with variable amounts of anti-T4 mAb or anti-T3 mAb (0-300 ng) in a total volume of $400 \mu \mathrm{l}$ PBS-BSA. Incubation was carried out for $1 \mathrm{~h}$ at $37^{\circ} \mathrm{C}$ and immune complexes were collected using sheep anti-mouse IgG immobilized on activated silica beads. After $20 \mathrm{~min}$ incubation at room temperature, beads were pelleted by centrifugation at $1500 \mathrm{~g}$ for $30 \mathrm{~min}$ at $4{ }^{\circ} \mathrm{C}$ and washed in PBS-BSA. Bound $\left[{ }^{125} \mathrm{I}\right] \mathrm{T} 4$ or $\left.{ }^{125} \mathrm{I}\right] \mathrm{T} 3$ was expressed as a percentage of the total radioactivity introduced into the assay. Each symbol represents the mean of duplicates. (B) and (C) Hormone binding specificity of mAbs. Competitive binding between labeled T4 or T3 and unlabeled T4, T3 or rT3. $\left[{ }^{125} \mathrm{l}\right] \mathrm{T} 4$ or $\left[{ }^{125} \mathrm{l}\right] \mathrm{T} 3(\approx 40000$ c.p.m.) was incubated with $30 \mathrm{ng}$ anti-T4 mAb (B) or $10 \mathrm{ng}$ anti-T3 mAb (C) and increasing concentrations of unlabeled hormone $(0-1000 \mathrm{nmol} / \mathrm{l})$ using the experimental conditions described above. Binding values, ' $\mathrm{B}$ ', obtained for each concentration of competitor were expressed as a percentage of maximum binding, 'Bo', obtained in the absence of competitor. Symbols are the means of duplicates in a representative experiment. in solution. Three purified hTgRP, with different iodine content, were used as competitor in the RIA; results are reported in Fig. 2. Each of the three purified Tg induced a concentration-dependent displacement of the binding of (i) labeled T4 to the anti-T4 mA b and (ii) labeled T3 to the anti-T3 mAb. As the competition curves were roughly parallel to the curves generated with unlabeled T4 or T3, it was possible to estimate the number of immunoreactive T4 or T3 residues on each purified Tg. Values are reported in Table 1. The higher the iodine content of $\mathrm{Tg}$, the higher the number of immunoreactive $\mathrm{T} 4$ residues. As far as T3 is concerned, the lowiodinated Tg, hTgRP4 (two iodine atoms/molecule), exhibited about the same immunoassayable T3 content as the most iodinated Tg, hTgRP8 (20 iodine atoms/ molecule).

\section{Adsorption of Tg onto immobilized anti-hTg mAb}

Data in Fig. 3 show that purified $\mathrm{Tg}$ (from the three reference preparations), diluted in serum So, and serum
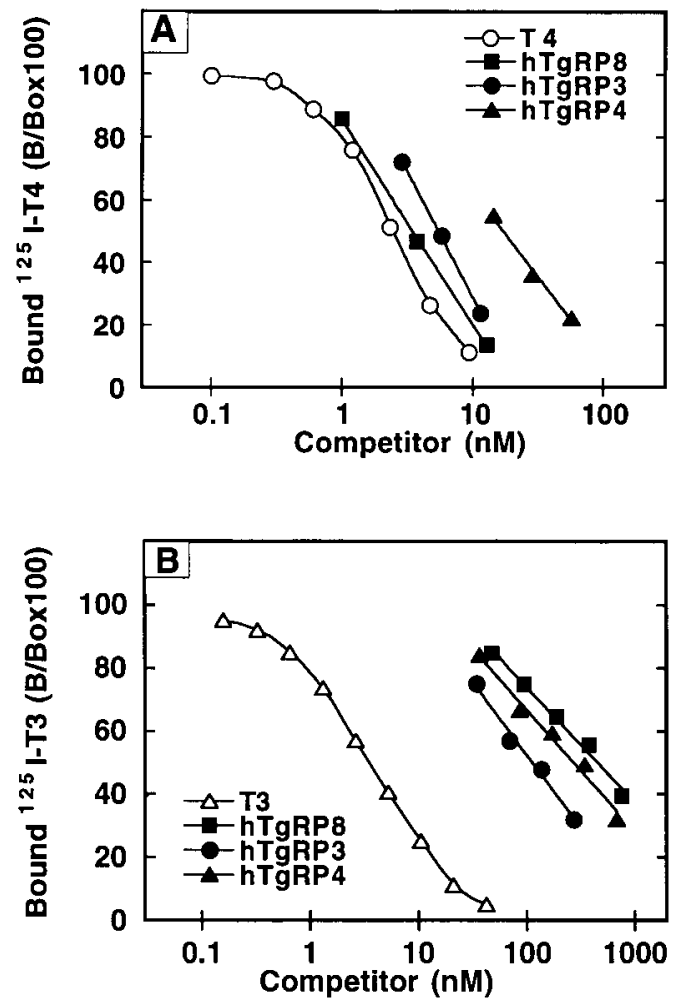

Figure 2 Capacity of the anti-T4 and anti-T3 mAbs to detect hormone residues on $\mathrm{Tg}$ in solution. Displacement of the binding of labeled T4 to the anti-T4 mAb (A) and of labeled T3 to the anti-T3 $\mathrm{mAb}(\mathrm{B})$ by purified Tg. $\left[{ }^{125} \mathrm{l}\right] \mathrm{T} 4$ or $\left[{ }^{125}\right] \mathrm{T} 3$ was incubated with $30 \mathrm{ng}$ anti-T4 mAb or $10 \mathrm{ng}$ anti-T3 mAb and increasing concentrations of unlabeled hormone (T4 or T3) or purified Tg (hTgRP3, hTgRP4 and hTgRP8). Incubation and measurements were performed as indicated in the legend of Fig. $1 \mathrm{~A}$. 
Table 1 Detection of thyroid hormone residues on purified Tg using anti-T4 and anti-T3 mAbs. The T4 and T3 content of three purified Tg preparations, hTgRP3, hTgRP4 and hTgRP8 was evaluated by (i) competitive binding between T4 or T3 and soluble Tg in RIA using the anti-T4 mAb or the anti-T3 mAb (Fig. 2) and (ii) mAb binding assays on adsorbed Tg (Fig. 4). Results are expressed in moles T4 or moles T3 per mole $\mathrm{Tg}$ and presented as the mean \pm S.E.M. of the values obtained with three quantities of $\mathrm{Tg}$ in at least two different assays. T4 and T3 values obtained by mAb binding assays were calculated from the labeled anti-T4 mAb and labeled anti-T3 mAb binding values and the known specific radioactivity of the labeled mAbs; it was assumed that one mAb molecule reacts with one hormone residue.

\begin{tabular}{|c|c|c|c|c|c|}
\hline \multirow[b]{3}{*}{ hTg } & \multirow{3}{*}{$\begin{array}{c}\text { lodine content } \\
\text { (atoms/Tg molecule) }\end{array}$} & \multicolumn{4}{|c|}{ Hormone content of $\mathrm{Tg}$ (mole/mole $\mathrm{Tg}$ ) determined by } \\
\hline & & \multicolumn{2}{|c|}{ RIA } & \multicolumn{2}{|c|}{ mAb binding assay } \\
\hline & & $\mathrm{T} 4$ & T3 & $\mathrm{T} 4$ & T3 \\
\hline RP4 & 2 & $0.14 \pm 0.01$ & $0.014 \pm 0.002$ & $0.012 \pm 0.002$ & $0.0031 \pm 0.0004$ \\
\hline RP3 & 8 & $0.52 \pm 0.07$ & $0.029 \pm 0.003$ & $0.029 \pm 0.013$ & $0.0071 \pm 0.0004$ \\
\hline RP8 & 20 & $0.66 \pm 0.06$ & $0.011 \pm 0.001$ & $0.036 \pm 0.001$ & $0.0032 \pm 0.0003$ \\
\hline
\end{tabular}
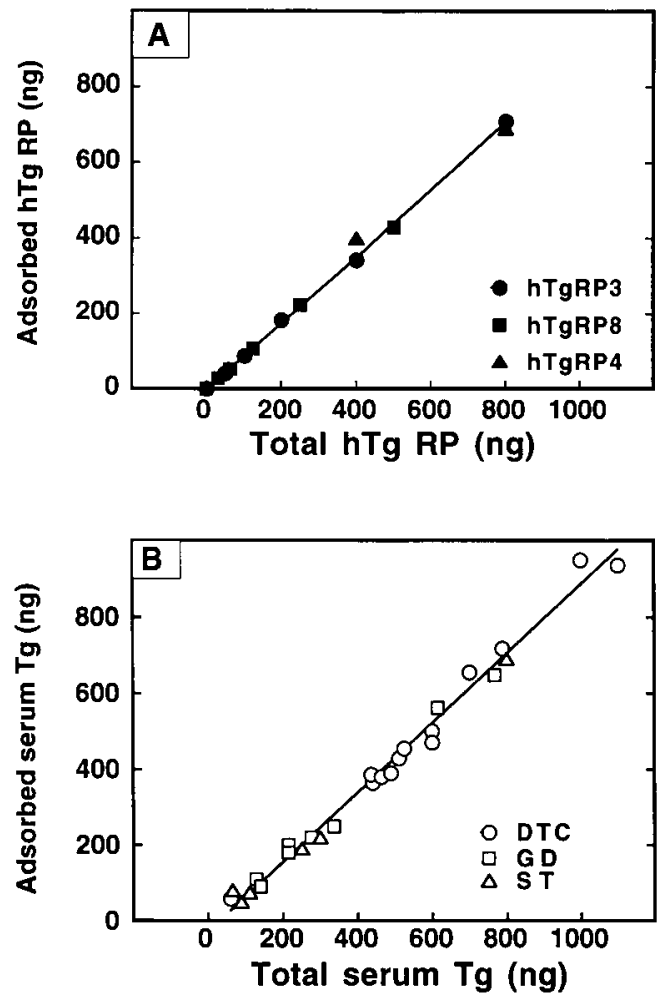

Figure 3 Adsorption of purified Tg and serum Tg on immobilized anti-hTg mAb. Various amounts of purified $\mathrm{Tg}(10-800 \mathrm{ng}$ of hTgRP3, hTgRP4 or hTgRP8) in $1 \mathrm{ml}$ serum So (A) or serum $\mathrm{Tg}$ from DTC, GD or ST patients ( $30 \mu \mathrm{l}$ to $1 \mathrm{ml}$ serum according to $\mathrm{Tg}$ concentration) supplemented to $1 \mathrm{ml}$ with serum So (B) were incubated in anti-hTg mAb-coated tubes. After a $6 \mathrm{~h}$ incubation period at room temperature and overnight at $4{ }^{\circ} \mathrm{C}$, serum was removed and assayed for its residual $\mathrm{Tg}$ content by $\mathrm{Tg}$ RIA as described in Materials and methods. Adsorbed Tg was calculated as the difference between the amount of $\mathrm{Tg}$ introduced into the tube (total $\mathrm{Tg}$ ) and the amount of $\mathrm{Tg}$ recovered in the serum at the end of incubation (free $\mathrm{Tg}$ ).
Tg from either DTC, ST or GD patients were quantitatively retained on anti-hTg mAb-coated tubes. The percentage of adsorption of Tg varied from 85 to $90 \%$ over a wide range of concentrations $(10-1000 \mathrm{ng} / \mathrm{ml})$.

\section{Binding of labeled anti-T4 or anti-T3 mAbs to adsorbed Tg}

Binding of radiolabeled anti-T4 or anti-T3 $\mathrm{mA}$ bs to antihTg mAb-coated tubes, previously incubated (adsorption step) with serum So, was lower than or equal to $0.5 \%$. Radiolabeled $\mathrm{mAb}$ binding values significantly higher than the background level were obtained when 10-15 ng hTgRP3, hTgRP4 or hTgRP8 were added to serum So. To exclude a possible interference of serum thyroid hormones through the non-specific adsorption of serum proteins to the anti-hTg mAb-coated tubes, it was verified that neither $1 \mu \mathrm{mol} / \mathrm{l}$ T4 nor $1 \mu \mathrm{mol} / / \mathrm{T} 3$ added to serum albumin in PBS or to serum So (during the adsorption step) increased radiolabeled $\mathrm{mA} b$ binding over the background value.

As shown in Fig. 4, the binding of radiolabeled antiT4 mAb to adsorbed hTgRP3, hTgRP4 and hTgRP8 increased linearly with the amount of adsorbed Tg; the slope of the line was different for the three hTgRP, indicating that each purified Tg had a different number of T4 residues accessible to the mAb (Fig. 4). Similarly, the binding of radiolabeled anti-T3 mAb was proportional to the amount of adsorbed Tg. The binding of labeled anti-T4 $\mathrm{mAb}$ to $\mathrm{Tg}$ was specific since $\mathrm{a}$ preincubation of the labeled $\mathrm{mAb}$ with $100 \mathrm{nmol} / \mathrm{l}$ T4 totally prevented its binding to adsorbed Tg. A preincubation with $\mathrm{T} 3$ did not modify the interaction of the labeled anti-T4 mAb with Tg. Similarly, T3 but not $\mathrm{T} 4$, inhibited the binding of the labeled anti-T3 $\mathrm{mA} b$ to adsorbed Tg. From the binding values of Fig. $4 \mathrm{~A}$ and $\mathrm{B}$ and the known specific radioactivity of labeled $\mathrm{mA}$ bs, it was possible to calculate the number of anti-T4 $\mathrm{mAb}$ or anti-T3 mAb molecules bound per Tg molecule. There was a good relationship between the number of anti-T4 $\mathrm{mAb}$ or anti-T3 mAb molecules bound to adsorbed Tg 

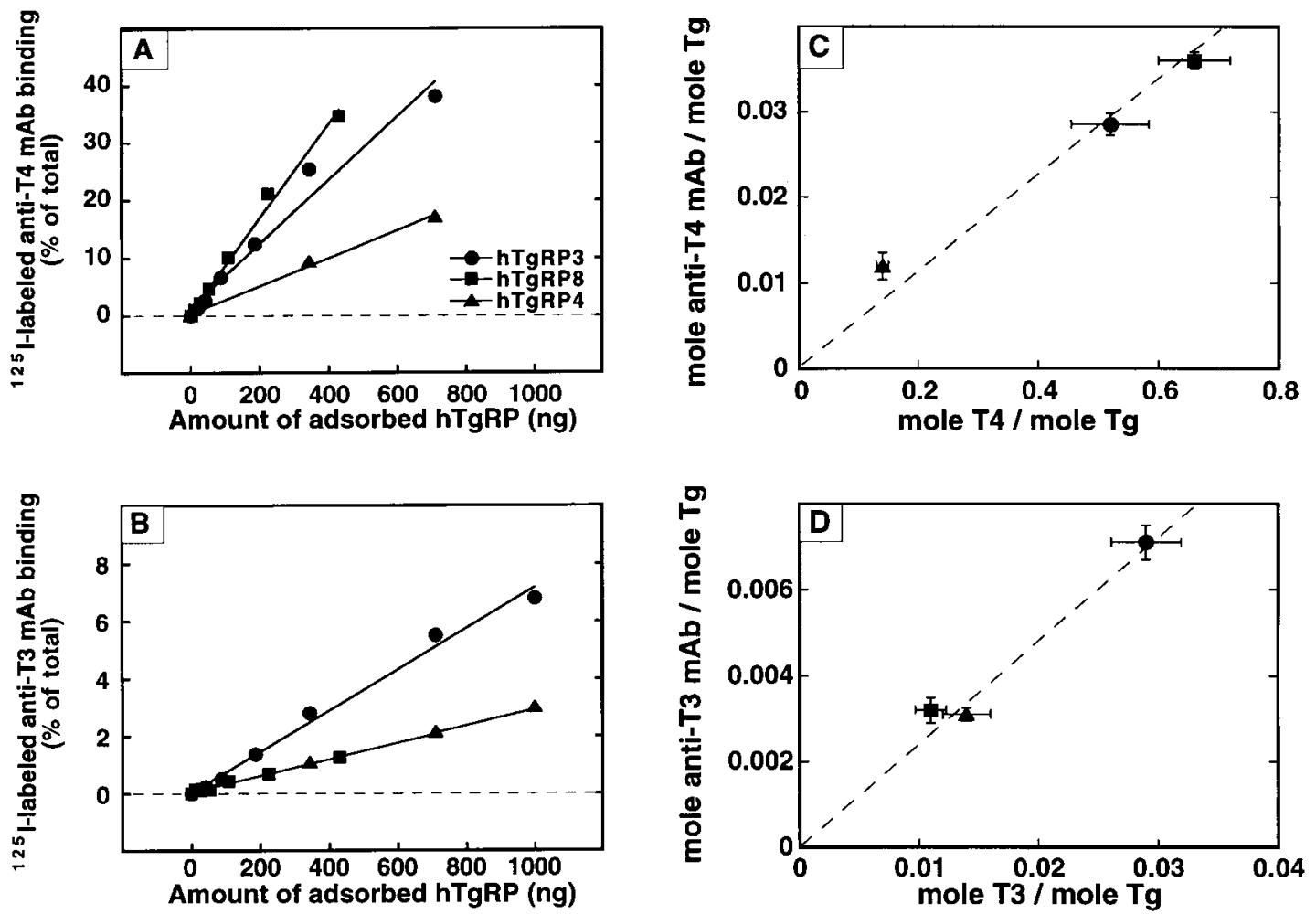

Figure 4 Detection of thyroid hormone residues on adsorbed Tg by mAb binding assay. Binding of labeled anti-T4 mAb (A) or labeled anti-T3 mAb (B) as a function of the amount of adsorbed purified Tg. About 100000 c.p.m. of either ${ }^{125}$-labeled anti-T4 mAb (67 fmol) or ${ }^{125}$ I-labeled anti-T3 $\mathrm{mAb}(155 \mathrm{fmol})$ were added to tubes containing various amounts $(0-1000 \mathrm{ng})$ of adsorbed hTgRP3, hTgRP4 or hTgRP8. Incubation and measurements were performed as described in the Materials and methods section. Binding of ${ }^{125} \mathrm{I}-\mathrm{labeled} \mathrm{mAbs}$ to adsorbed Tg was expressed as a percentage of total radioactivity. (C) and (D) Relationship between the anti-T4 mAb binding (C) or the anti-T3 mAb binding (D) to adsorbed Tg and the number of T4 or T3 residues detected on Tg in solution by RIA. The mAb binding values reported in panels $A$ and $B$ were expressed in moles anti-T4 mAb or moles anti-T3 mAb bound per mole $\mathrm{Tg}$ and plotted versus the number of T4 or T3 residues detected on each hTgRP by RIA (Table 1). Symbols represent the mean, and vertical and horizontal bars the S.E.M. of the values $(n=6-8)$ obtained by mAb binding assay and RIA respectively.

(mole per mole $\mathrm{Tg}$ ) and the number of $\mathrm{T} 4$ or $\mathrm{T} 3$ residues (mole per mole Tg), disclosed on Tg in solution by RIA (Fig. 4C and D). Considering that one antibody molecule reacts with one hormone residue, the number of $\mathrm{mAb}$ molecules bound per $\mathrm{Tg}$ molecule was converted into the number of $\mathrm{T} 4$ or $\mathrm{T3}$ residues detected per $\mathrm{Tg}$ molecule; values are reported in Table 1 . About $6 \%$ of the T4 residues and $25 \%$ of the T3 residues detected on $\mathrm{Tg}$ in solution by RIA did react with $\mathrm{mA}$ bs on adsorbed Tg.

\section{Identification of hormone residues on serum Tg from patients with thyroid disorders}

Serum Tg from 27 patients (8 GD, 13 DTC, 6 ST) was analyzed for its capacity to bind labeled mAbs after adsorption onto the immobilized anti-hTg mAb. The average amount of adsorbed Tg from GD, DTC and ST sera, subjected to the $\mathrm{mAb}$ binding assays, was $280 \pm$ $73,465 \pm 59$ and $217 \pm 99 \mathrm{ng}$ (mean \pm S.E.M., $\mathrm{n}=6-$ 13) respectively; there was no statistically significant difference between the three groups. The binding of labeled anti-T4 mAb and labeled anti-T3 mAb to each serum Tg sample is shown in Fig. 5. With one exception, serum $\mathrm{Tg}$ from patients with GD did not have detectable anti-T4 mAb or anti-T3 mAb binding activity. The patient whose Tg exhibited a low but significant anti-T4 $\mathrm{mAb}$ binding activity did not present any biological or clinical peculiarity as compared with other patients with GD. Out of 13 patients with DTC, only 1 had serum $\mathrm{Tg}$ molecules capable of interacting with anti-T4 and anti-T3 mAbs. This case (patient DTC 89) is presented below. In contrast, all of six sera from patients with ST contained $\mathrm{Tg}$ with anti-T4 $\mathrm{mAb}$ and anti-T3 mAb binding activities. Data in Fig. 6 allow a comparison of the apparent T4 and T3 contents of serum $\mathrm{Tg}$ and purified Tg. In seven of the eight serum samples that were positive in the $\mathrm{mA} b$ binding assay, the apparent T4 content of serum $\mathrm{Tg}$ was close to that of hTgRP4 characterized by a low iodine content. In one case, the apparent T4 content of serum Tg exceeded that of the most iodinated reference Tg, hTgRP8 containing 20 iodine atoms/molecule. Serum $\mathrm{Tg}$ samples that were positive in the anti-T3 mAb binding assay showed an 

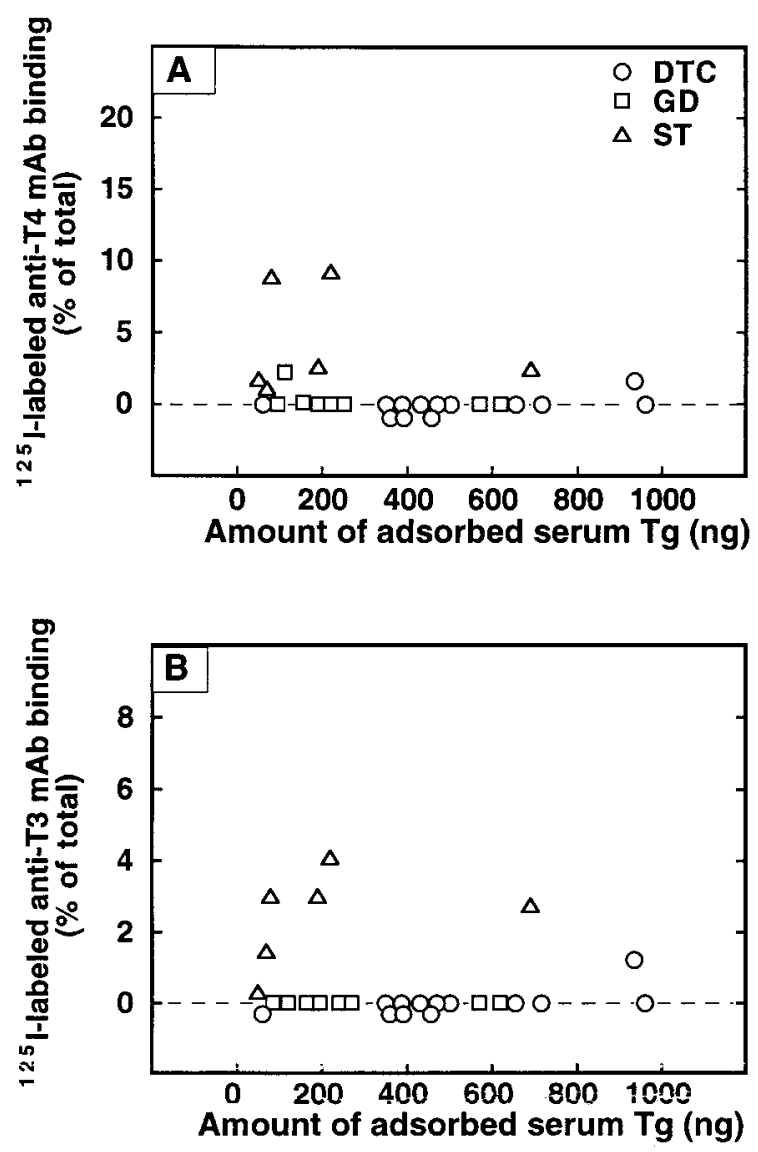

Figure 5 Detection of thyroid hormone residues on adsorbed $\mathrm{Tg}$ from human sera by mAb binding assays. Tg present in the serum of 8 patients with GD, 13 with DTC and 6 with ST was adsorbed on the immobilized anti-hTg mAb (see Fig. 3) and incubated with labeled anti-T4 mAb (A) or labeled anti-T3 mAb (B) in the conditions described in the legend of Fig. 4. Labeled mAb binding, expressed as a percentage of total radioactivity, was plotted versus the amount of adsorbed Tg. Symbols represent the mean of duplicate or triplicate measurements on individual sera.

apparent T3 content equal to or higher than that of the three purified $\mathrm{Tg}$.

\section{Analyses of the T4 and T3 content of serum Tg from a patient with thyroid hormone producing metastases. Effect of antithyroid drug therapy}

Four serum samples from patient DTC 89 were analyzed for their anti-T4 $\mathrm{mAb}$ and anti-T3 $\mathrm{mAb}$ binding activities (Fig. 7). The first serum sample (6-89) (corresponding to the positive serum in the group of patients with DTC - Figs 5 and 6) presented a very high $\mathrm{Tg}$ concentration and low but significant anti-T4 mAb and anti-T3 $\mathrm{mAb}$ binding activities. At that time, the patient, who was hyperthyroid, was placed under methimazole treatment. Four months later (10-89), serum $\mathrm{Tg}$ concentration was still elevated but the apparent T4 and T3 content of serum Tg was decreased by $90 \%$. As the patient was euthyroid, the treatment was stopped and 2 months later (12-89), there was a marked elevation of the apparent T4 and T3 content of serum Tg. From that time on, only one serum sample could be analyzed. Serum Tg from the 4-94 sample was devoid of $m A b$ binding activity. At that time, the patient had received a total dose of $700 \mathrm{mCi}$ and there were no more sites of radioiodide uptake.

\section{Discussion}

Using $\mathrm{mAbs}$, an anti-hTg mAb immobilized on a solid phase and radiolabeled anti-T4 or anti-T3 mAbs, we developed a procedure for probing the presence of thyroid hormone residues on minute amounts of $\mathrm{Tg}$ in unfractionated human serum. The two steps of the method, (i) quantitative adsorption or capture of $\mathrm{Tg}$ and (ii) hormone residue recognition by specific $\mathrm{mA}$ bs, have been validated using purified hTg preparations. The adsorption of $\mathrm{Tg}$, through an immobilized anti-hTg $\mathrm{mAb}$, was quantitative for both purified Tg and Tg from the serum of patients with different thyroid disorders. This was not unexpected since this anti-hTg mAb is currently used in a commercialized immunoradiometric $\mathrm{Tg}$ assay. The anti-T4 and anti-T3 mA bs, which show a good specificity for either T4 or T3, selectively recognized T4 and T3 residues on $\mathrm{Tg}$ in solution and on adsorbed Tg. Serum thyroid hormones, either free or bound to serum proteins, did not interfere in the assay.

Detection of thyroid hormone residues on Tg was only semi-quantitative. The yield of T4 residue detection on $\mathrm{Tg}$ in solution (assessed by competitive binding using the anti-T4 mAb in RIA) was about $30 \%$ for hTgRP8 (20 iodine atoms/molecule), $50 \%$ for hTgRP3 (8 iodine atoms/molecule) and probably near to $100 \%$ for $\mathrm{hTgRP} 4$ (2 iodine atoms/molecule), assuming that hTgRP4 had an average T4 content of 0.15 residue per molecule. This value was extrapolated from data of Rolland et al. (1) who reported that low-iodinated $\mathrm{Tg}$ (one to three iodine atoms/molecule) contains $0.1-0.2 \mathrm{~T} 4$ residue/ molecule. It appears thus that the lower the iodine content, the higher the yield of detection of T4 residues. This finding is in keeping with previous data from Byfield et al. (11) who studied the capacity of polyclonal antibodies raised against a T4-albumin conjugate to react with $\mathrm{hTg}$. The yield of detection of $\mathrm{T} 3$ on $\mathrm{Tg}$ in solution could not be estimated since the actual T3 content of purified Tg preparations could not be accurately measured.

Several factors may contribute to the variability and lowering of the yield of detection of hormone residues on intact Tg in solution. Individual T4 or T3 residues on $\mathrm{Tg}$ could vary in their accessibility and/or reactivity with $m A b s$. The influence of the three dimensional structure of $\mathrm{Tg}$ on the ability of $\mathrm{mA}$ bs to detect hormone residues on Tg has already been reported (12). Steric hindrance to the approach of a second immunoglobulin after the 

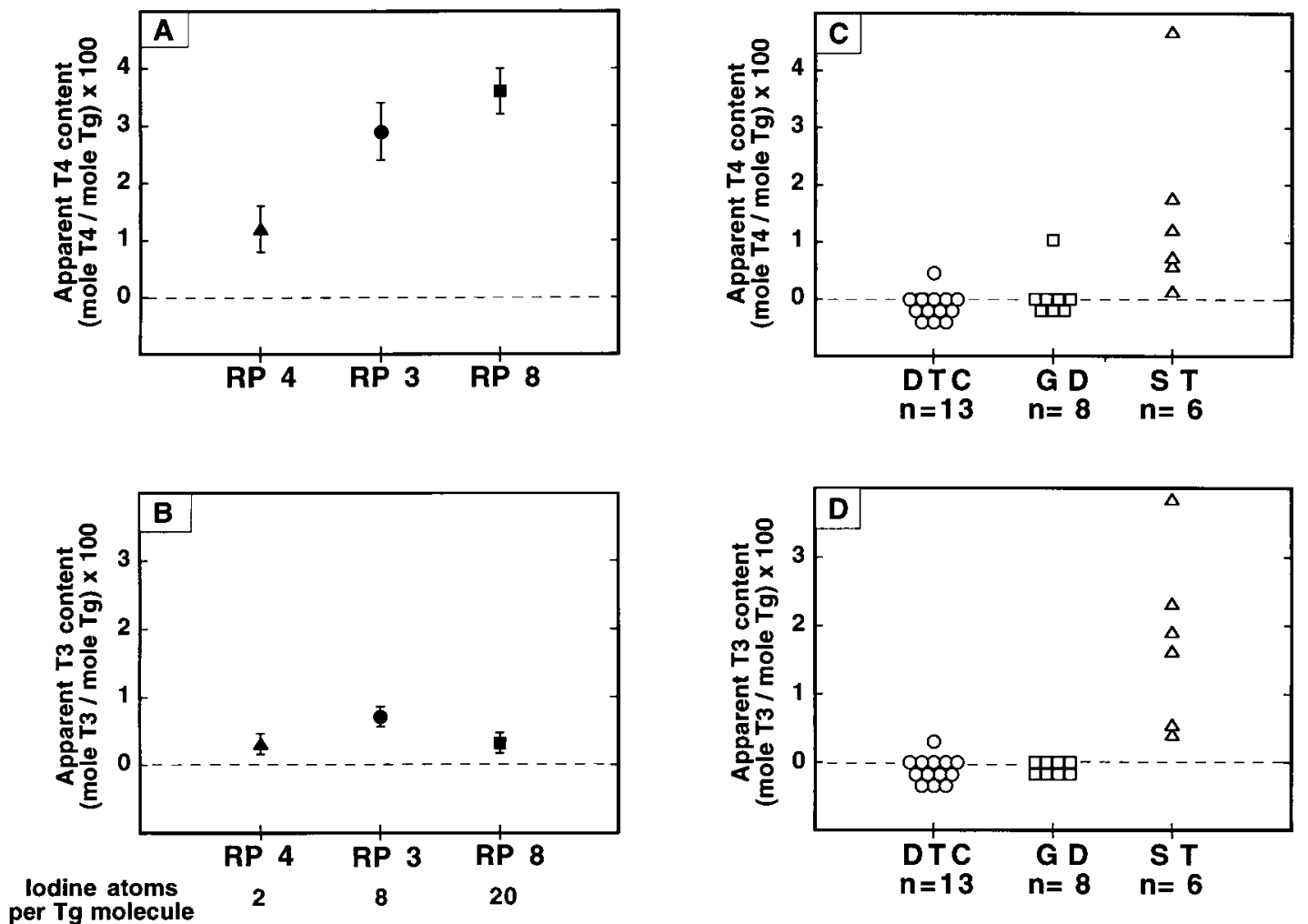

Figure 6 Apparent T4 and T3 content of Tg from the serum of patients with DTC, GD or ST (C and D) deduced from the mAb binding assays. Comparison with the apparent thyroid hormone content of purified Tg preparations with different iodine content (A and B). Values of $\mathrm{T} 4$ and T3 content of hTgRP3, hTgRP4 and hTgRP8 were taken from Table 1. The apparent T4 and T3 content of serum Tg was calculated from the labeled anti-T4 mAb and labeled anti-T3 mAb binding values presented in Fig. 5 and the known specific radioactivity of labeled mAbs. It was assumed that one mAb molecule reacts with one hormone residue.

first has bound might represent another limitation for the detection of $\mathrm{T} 4$ residues on more iodinated $\mathrm{Tg}$ molecules.

The capacity of anti-T4 mAb and anti-T3 mAb to react with purified $\mathrm{Tg}$, once adsorbed onto a solid phase, was markedly lowered but to about the same extent for each purified Tg preparation. This indicates that immobilization of $\mathrm{Tg}$ causes additional constraints in the reactivity and/or accessibility of hormone residues to mAbs; these constraints were much more pronounced for T4 than for T3. Indeed, the yield of T4 detection on adsorbed $\mathrm{Tg}$ was decreased about 16 -fold as compared with the yield of $\mathrm{T} 4$ detection on $\mathrm{Tg}$ in solution, whereas the adsorption of $\mathrm{Tg}$ only led to a 4 -fold reduction in the T3 detection yield.

Although not quantitative, $\mathrm{mAb}$ binding assays provide parameters related to the actual hormone content of $\mathrm{Tg}$. First, the number of $\mathrm{T} 4$ residues uncovered on purified $\mathrm{Tg}$ by the mAb binding assay increased with the iodine content of Tg. Secondly, as expected from literature data $(13,14)$, more T3 (as compared with T4) was detected on Tg with a low iodine content than on $\mathrm{Tg}$ with a high iodine content. Despite its low yield, the method of hormone residue detection on adsorbed $\mathrm{Tg}$ presents a good sensitivity.
Indeed, T4 and T3 residues were readily detected from $40 \mathrm{ng}$ of the low-iodinated Tg, hTgRP4. Considering that hTgRP4 (two iodine atoms/molecule) contained, at the very most, $25 \%$ of $\mathrm{Tg}$ molecules with one hormone residue (1), one can estimate that, starting from $200-$ $400 \mathrm{ng}$ of adsorbed $\mathrm{Tg}$, the $\mathrm{mAb}$ binding assays could detect Tg molecules (with at least one hormone residue) accounting for not more than $2-5 \%$ of total Tg. Thus, patient sera with an immunoreactive $\mathrm{Tg}$ concentration equal to or higher than $200 \mathrm{ng} / \mathrm{ml}$ that were negative in the mAb binding assays, were probably devoid of or contained less than $5 \%$ of $\mathrm{Tg}$ molecules with thyroid hormone residues. This is the case for 16 out of 21 sera from patients with either DTC or GD. Among the four sera with a lower Tg concentration, one was clearly positive in the anti-T4 $\mathrm{mAb}$ binding assay; the presence of $\mathrm{Tg}$ with hormone residues in the three other sera cannot be excluded.

The presence of thyroid hormone residues on serum Tg from patients with ST brings experimental support to previously made hypotheses about the source and the mode of release of $\mathrm{Tg}$ appearing in the circulation in this disease. In the acute phase of the inflammatory process, $\mathrm{Tg}$ molecules that have undergone hormonogenesis in the lumen of thyroid follicles are probably released into 


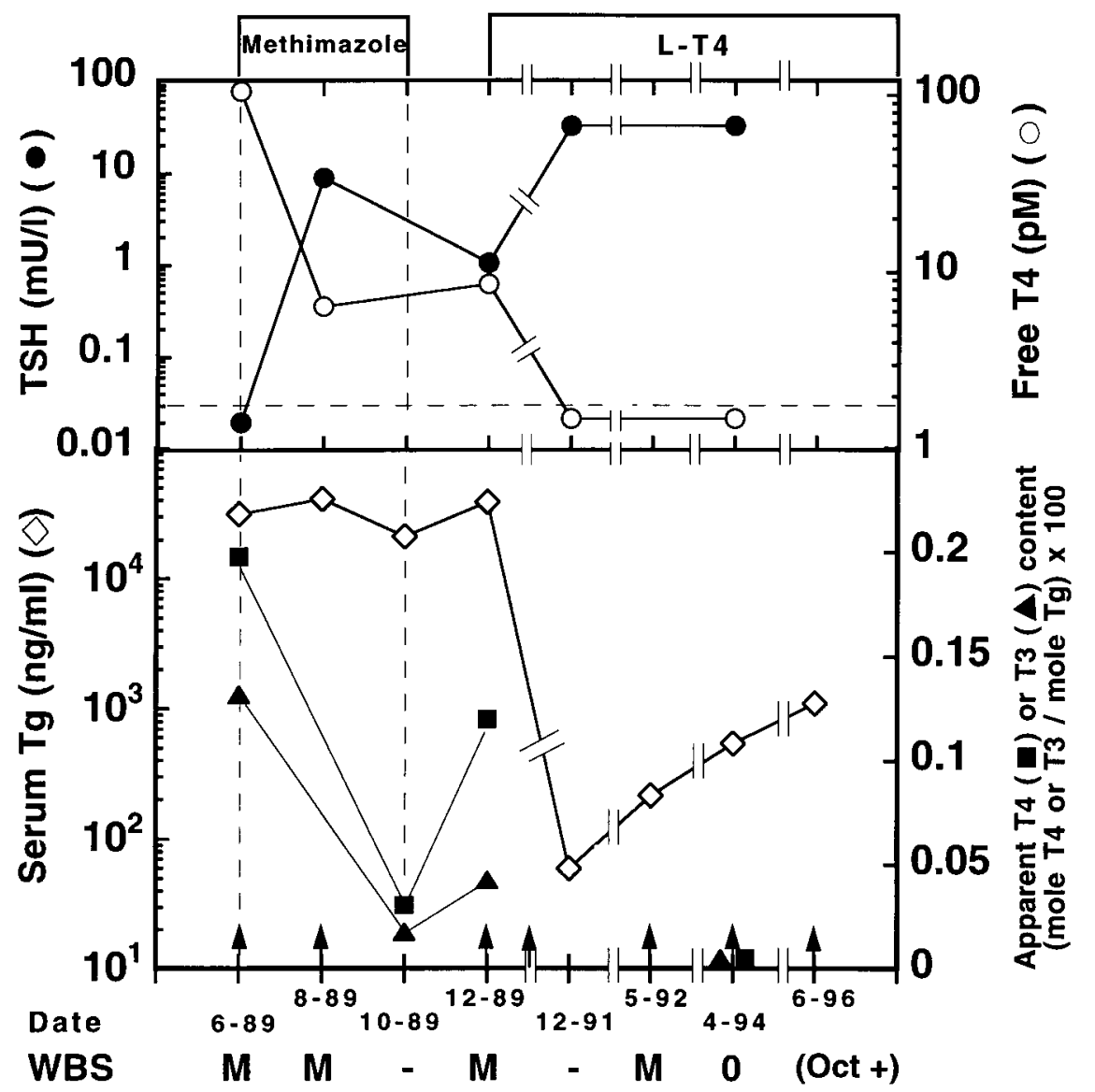

Figure 7 Sequential measurements of serum TSH, serum free T4, serum Tg and apparent T4 and T3 content of serum Tg in patient DTC 89. Date of serum sampling is indicated by two numbers corresponding to the month and the year. Arrows indicate the time of administration of therapeutic doses of ${ }^{131} \mathrm{I}$. The outcome of WBS after administration of $100 \mathrm{mCi}^{131} \mathrm{I}$ is given at the bottom of the figure (M: metastases disclosed by radioiodide uptake; 0: no radioiodide uptake). Oct+: metastases disclosed by Octreoscan. The 4 month period of treatment by methimazole and the period of treatment by L-T4 are indicated at the top of the figure.

the blood stream as the consequence of the rupture of the tightness of follicle lumena. Serum Tg from patients with ST was distinguishable from purified $\mathrm{Tg}$ by its apparent T3/T4 ratio, which was 3- to 10-fold higher than that of the three purified Tg. This could be due to particular alterations of $\mathrm{Tg}$ structure that would favor T3 recognition and/or minimize T4 detection. Alternately, in the course of their exit from the follicles, $\mathrm{Tg}$ molecules might be subjected to proteolytic reactions leading to cleavage of $\mathrm{T} 4$ residues. The latter hypothesis is in keeping with previous data (9) showing that immunoreactive serum $\mathrm{Tg}$, identified by Western blotting, decreased after reduction of disulfide bonds.

We have been unable (except in one case) to detect hormone residues on serum Tg from patients with GD. This was unexpected considering previous studies in human $(4-6)$ and in rat $(15,16)$ suggesting that $\mathrm{Tg}$ released by the stimulated thyroid gland would mainly correspond to iodinated $\mathrm{Tg}$ molecules, thus originating from the lumen of thyroid follicles. From physicochemical analyses of serum $\mathrm{Tg}$ by velocity sedimentation on sucrose gradients and Western blotting (9), we recently proposed a pathway of $\mathrm{Tg}$ release that could explain the lack of T3 and T4 residues on serum $\mathrm{Tg}$ from patients with GD. Tg molecules internalized at the apical pole of thyrocytes, conveyed to early endosomes (17) and subjected to apical-basolateral transcytosis (3) could undergo, during their transcellular transport, discrete proteolytic cleavage reactions that could generate hormone-depleted molecules. It has been demonstrated (18) that removal of T4 and T3 residues from $\mathrm{Tg}$ can occur without major alterations of $\mathrm{Tg}$ structure. $\mathrm{Tg}$ from the GD serum which was positive in the anti-T4 $\mathrm{mAb}$ binding assay could represent a case of incomplete thyroid hormone removal. Studies on a larger number of GD sera are necessary to determine whether the presence of $\mathrm{Tg}$ with hormone residues is incidental or represents a more frequent situation.

Our inability to detect hormone residues on serum $\mathrm{Tg}$ from patients with DTC further supports the idea that $\mathrm{Tg}$ released by metastatic thyroid carcinoma mostly, if not exclusively, corresponds to non-iodinated molecules 
(19). The patient DTC 89, whose serum Tg was found to contain T4 and T3 residues, is an exceptional case in many respects. According to the review of Paul \& Sisson (20), less than 20 cases of metastatic thyroid cancer causing thyrotoxicosis have been reported. In the present patient, the production of thyroid hormones by distant metastases was very high; free T4 concentration was about 5 -fold higher than the upper limit of the normal range. Tg production by metastases was also extremely high. Using metabolic parameters of serum Tg determined by Lo Gerfo et al. (21) in patients with DTC, one can estimate that the maintenance of serum $\mathrm{Tg}$ at a value of $30 \mu \mathrm{g} / \mathrm{ml}$ (serum Tg concentration of patient DTC 89) would require a daily production of about $165 \mathrm{mg}$. By comparison, the amount of $\mathrm{Tg}$ synthesized per day by a normal thyroid gland is about $30 \mathrm{mg}$ (22). The high serum T4 and serum Tg concentrations were related to the existence of large tumor masses with differentiated thyroid activities. The presence of $\mathrm{T} 4$ and $\mathrm{T} 3$ residues on serum $\mathrm{Tg}$ from patient DTC 89 indicates that part of the circulating $\mathrm{Tg}$ was coming from follicular structures where iodination and coupling reactions take place. This is further shown by the fact that methimazole treatment that efficiently restored euthyroidism caused an almost complete disappearance of serum $\mathrm{Tg}$ with hormone residues.

The identification of thyroid hormone residues on circulating $\mathrm{Tg}$, in diverse pathophysiological situations, supports the idea that circulating $\mathrm{Tg}$ may serve as substrate for extrathyroidal production of thyroid hormones. The group of Herzog $(23,24)$ recently reported that macrophages in culture are capable of releasing $\mathrm{T} 4$ and $\mathrm{T} 3$ from $\mathrm{Tg}$, a process requiring the internalization and then the proteolytic processing of Tg. Furthermore, these authors suggest that T3 and T4 produced by Kupffer cells of the liver could directly act on neighboring hepatocytes. To have a biological significance, this production of thyroid hormones would require elevated serum $\mathrm{Tg}$ concentration and/or serum $\mathrm{Tg}$ with an elevated hormonal content. There are probably only a few pathological situations in which these conditions are fulfilled. In patients with ST, serum $\mathrm{Tg}$ at a concentration $\geq 1000 \mathrm{ng} / \mathrm{ml}$ (as was the case in two patients of this study) possessing one or two T4 residues per molecule, could generate an amount of $\mathrm{T} 4$ accounting for more than $10 \%$ of the average daily normal production of T4 $(80 \mu \mathrm{g} /$ day). It is thus reasonable to think that, in some patients with ST, the extrathyroidal production of T4 may contribute to the transient thyrotoxicosis that characterizes the acute phase of the disease (25).

\section{Acknowledgements}

We thank Prof. F Berthezene and Dr F Borson-Chazot (Service d'Endocrinologie, Hôpital de l'Antiquaille, Lyon), Prof. J Orgiazzi (Service de Médecine Interne, Centre Hospitalier Lyon-Sud), Prof. J P Riou (Service
d'Endocrinologie, Hôpital Edouard-Herriot, Lyon), Prof. J Tourniaire (Clinique Endocrinologique, Hôpital de I'Antiquaille, Lyon), and $\operatorname{Dr} M$ D'Herbomez (Centre Hospitalier Régional et Universitaire, Lille) for providing us with clinical information concerning the patients whose sera were used in these studies. We are grateful to M P Seguin from Cis bio international for providing us with anti-hTg mAb-coated tubes and purified anti-T4 and anti-T3 mAbs.

\section{References}

1 Rolland M, Montfort MF, Valenta L \& Lissitzky S. Iodoamino acid composition of the thyroglobulin of normal and diseased thyroid glands: comparison with in vitro iodinated thyroglobulin. Clinica Chimica Acta 197239 95-108.

2 Chambard M, Mauchamp J \& Chabaud O. Synthesis and apical and basolateral secretion of thyroglobulin by thyroid cell monolayers on permeable substrate: modulation by thyrotropin. Journal of Cellular Physiology 1987133 37-45.

3 Herzog V. Transcytosis in thyroid follicle cells. Journal of Cell Biology 198397 607-617.

4 Uller RP, Van Herle AJ \& Chopra IJ. Comparison of alterations in circulating thyroglobulin, triiodothyronine and thyroxine in response to exogenous (bovine) and endogenous (human) thyrotropin. Journal of Clinical Endocrinology and M etabolism $197337741-745$.

5 Unger J, Van Heuverswyn B, Decoster C, Cantraine F, Mockel J \& Van Herle $A$. Thyroglobulin and thyroid hormone release after intravenous administration of bovine thyrotropin in man. Journal of Clinical Endocrinology and M etabolism 198051 590-594.

6 Uller RP \& Van Herle AJ. Effect of therapy on serum thyroglobulin levels in patients with Graves' disease. Journal of Clinical Endocrinology and M etabolism 197846 747-755.

7 Gentile F, Palumbo G\& Salvatore G. The origin of the electrophoretic doublet of thyroglobulin. Biochemical and Biophysical Research Communications 1992186 1185-1191.

8 Sandell EB \& Kolthoff IM. Micro determination of iodine by a catalytic method. Microchimica Acta 1937 1 9-15.

9 Druetta L, Croizet K, Bornet $H \&$ Rousset B. Analyses of the molecular forms of serum thyroglobulin from patients with Graves' disease, subacute thyroiditis or differentiated thyroid cancer by velocity sedimentation on sucrose gradient and western blot. European Journal of Endocrinology 1998139 498-507.

10 Feldt-Rasmussen $U$, Profilis C, Colinet $E$, Black E, Bornet $H$, Bourdoux $P$ et al. Human thyroglobulin reference material (CRM 457) 2nd part: physicochemical characterization and certification. Annales de Biologie Clinique 199654 343-348.

11 Byfield PGH, Bond A, Copping $S \&$ Himsworth RL. Restricted antigenicity of thyroxyls in human thyroglobulin. Biochemical Journal 1982207 471-478.

12 De Baets MH, Theunissen R, Kok K, De Vijlder JJM \& Van Breda Vriesman PJC. Monoclonal antibodies to human thyroglobulin as probes for thyroglobulin structure. Endocrinology $1987 \quad 120$ 1104-1111.

13 Studer $H \&$ Greer MA. A study of the mechanisms involved in the production of iodine deficiency goiter. Acta Endocrinologica 1965 $49610-628$.

14 Izumi M \& Larsen PR. Triiodothyronine, thyroxine, and iodine in purified thyroglobulin from patients with Graves' disease. Journal of Clinical Investigation 197759 1105-1112.

15 Schneider AB, Fleischmann $K \&$ Chu L. Thyrotropin increases the iodine content of rat circulating thyroglobulin as measured by equilibrium density gradient centrifugation. Biochimica et Biophysica Acta $1985838329-334$.

16 Schneider AB, Line B, Goldman | \& Robbins |. Sequential serum thyroglobulin determination, ${ }^{13} 1_{\mid}$scans, and ${ }^{131} 1_{\mid}$uptakes after triiodothyronine withdrawal in patients with thyroid cancer. 
Journal of Clinical Endocrinology and M etabolism 1981531199 1206.

17 Kostrouch Z, Munari-Silem Y, Rajas F, Bernier-Valentin F \& Rousset B. Thyroglobulin internalized by thyrocytes passes through early and late endosomes. Endocrinology $1991 \mathbf{1 2 9}$ 2202-2211.

18 Rousset B, Selmi S, Bornet H, Rabilloud R \& Munari-Silem Y Thyroid hormone residues are released from thyroglobulin with only limited alteration of the thyroglobulin structure. Journal of Biological Chemistry 1989264 12620-12626.

19 Schneider AB, Ikekubo K \& Kuma K. Iodine content of serum thyroglobulin in normal individuals and patients with thyroid tumors. Journal of Clinical Endocrinology and M etabolism 198357 1251-1256.

20 Paul SJ \& Sisson JC. Thyrotoxicosis caused by thyroid cancer. Endocrinology and M etabolism Clinics of North America 199019 593-612.

21 Lo Gerfo P, Colacchio T, Colacchio D \& Feind C. Serum clearance rates of immunologically reactive thyroglobulin. Cancer 197842 164- 166.
22 Izumi M, Kubo I, Taura M, Yamashita S, Morimoto I, Ohtakara S et al. Kinetic study of immunoreactive human thyroglobulin. Journal of Clinical Endocrinology and M etabolism 198662410 412.

23 Brix K \& Herzog V. Extrathyroidal release of thyroid hormones from thyroglobulin by $J 774$ mouse macrophages. Journal of Clinical Investigation 199493 1388-1396.

24 Brix K, Wirtz R \& Herzog V. Paracrine interaction between hepatocytes and macrophages after extrathyroidal proteolysis of thyroglobulin. Hepatology $1997261232-1240$.

25 Smallridge RC, De Keyser FM, Van Herle AJ, Butkus NE \& Wartofsky $L$. Thyroid iodine content and serum thyroglobulin: clues to the natural history of destruction-induced thyroiditis. Journal of Clinical Endocrinology and M etabolism 198662 12131219.

Received 28 August 1998

Accepted 4 January 1999 